\title{
Long term outcome and elasticity of a polyester mesh used for laparoscopic ventral hernia repair
}

\author{
P. J. O'Dwyer ${ }^{1} \cdot$ C. Chew ${ }^{2} \cdot$ H. Qandeel ${ }^{3}$
}

Received: 19 May 2021 / Accepted: 1 August 2021 / Published online: 23 August 2021

(c) The Author(s) 2021

\begin{abstract}
Background Repair of a ventral hernia is increasingly being performed by a laparoscopic approach despite lack of good long term follow up data on outcomes. The aim of this study was to examine the long term performance of a polyester mesh and to assess its elastic properties in patients undergoing laparoscopic ventral hernia repair.

Methods All patients being assessed for a ventral hernia repair between August 2011 and November 2013 were placed on a prospective database. Those undergoing laparoscopic repair with a polyester mesh were seen at clinic at one month and one year, while their electronic records were assessed at 34 months (range 24-48 months) and 104 months (range 92-116 months). In addition, $\mathrm{CT}$ scans of the abdomen and pelvis performed for any reason on these patients during the follow up period were reviewed by a consultant gastrointestinal radiologist. Mechanical failure testing of the mesh was also performed.

Results Thirty-two of the 100 patients assessed for ventral hernia repair had a laparoscopic repair with a polyester mesh. Nineteen (59\%) had CT scans performed during the follow-up period. No recurrence was recorded at 34 months, while three (9.4\%) had a recurrence at 104 months. Two had central breakdown of the mesh at 81 and 90 months, while 1 presented acutely at 116 months after operation. Mesh had stretched across the defect by an average of 21\% (range 5.7-40\%) in nine patients. Mechanical testing showed that this mesh lost its elasticity at low forces ranging between 1.8 and $3.2 \mathrm{~N} / \mathrm{cm}$.

Conclusion This study shows that late recurrence is a problem following laparoscopic ventral hernia repair with polyester mesh. The mesh loses it elasticity at a low force. This combined with degradation of mesh seems the most likely cause of failure. This is unlikely to be a unique problem of polyester mesh and further long-term studies are required to better assess this operative approach to ventral hernia repair.
\end{abstract}

Keywords Hernia $\cdot$ Laparoscopic $\cdot$ Mesh $\cdot$ Computed tomography $\cdot$ Late recurrence

\section{Introduction}

Repair of a ventral hernia is a common operation that is increasingly being undertaken by a laparoscopic approach [1]. The advantages of the laparoscopic over the open approach include shorter hospital stay, a more rapid return to normal activities and perhaps most importantly less wound complications. In a recent nationwide study of over 5000

P. J. O’Dwyer

Pjod2j@gmail.com

1 School of Medicine, Dentistry and Medicine, University of Glasgow, Glasgow, UK

2 Department of Radiology, University Hospital Hairmyres, Glasgow, UK

3 Department of Surgery, Hashemite University, Zarqa, Jordan patients recurrence rates were similar for open and laparoscopic repair after an average follow-up of 4 years [1]. This study also indicated that for defects between 2 and $6 \mathrm{~cm}$, the laparoscopic approach may be superior to open repair.

Most meshes are sufficiently strong to resist the pressures exerted on them by increased intraabdominal pressure such as with coughing. However, meshes degrade over time and lose their elasticity. A recent study has shown that a lightweight polyester mesh ruptured centrally when used to augment open ventral hernia repair [2]. A meta-analysis and systematic review of over 10,000 patients comparing polyester with polypropylene meshes, however, found no difference in recurrence rates between the products [3]. Follow-up in most of these studies was short with little data available on outcomes beyond 5 years.

The aim of this study was to examine the long-term performance of a polyester mesh and to assess its elastic 
properties in patients undergoing laparoscopic ventral hernia repair.

\section{Methods}

Between august 2011 and November 2013 all patients with a ventral hernia under the care of one surgeon were maintained on a prospective database. Data recorded included age, gender, Body mass index (BMI), American Society of Anaesthetist grade (ASA) type of hernia, defect width, clean or contaminated wounds, etc. Operative details including type of operation, mesh used, operative times and complications were also recorded, as were hospital stay and postoperative complications.

Patients considered suitable for a laparoscopic repair were those with a defect width of between 2 and $6 \mathrm{~cm}$ or those with high comorbidity irrespective of defect size. A monofilament polyester mesh with a hydrophilic porcine dermis collagen barrier (Parietex Composite Optimised Mesh, Covidien, New Haven, CT, USA) was used for all patients.

The abdominal cavity was accessed at Palmer's point using an optical port (Endopath Xcel, Ethicon). Two additional 5-mm ports were placed under direct vision and used to reduce the hernia and take down any adhesions present. An intraperitoneal onlay mesh was then inserted and placed across the defect with at least a 5-cm overlap. The mesh was secured with two rows of tacks (ProTack Covidien New Haven, CT, USA) and four transfacial sutures. An additional optical port and a 5-mm working port was also inserted on the patient's right side under direct vision. This allowed tacking from both sides and ensure the mesh was not lax following repair and overlap of defect was similar on both sides. All 10- to 12-mm port sites were closed with an absorbable suture.

Patients were followed-up at clinic at 1 month and 1 year while electronic notes were assessed at two further time points to access long-term outcomes. In addition, CT scans of the abdomen and pelvis performed for any reason during follow-up were reviewed by a consultant radiologist to determine if a recurrent hernia was present on CT. Also the mesh across the defect was measured on CT to determine if it had stretched during follow-up. Measurements were taken axial view where the defect was maximal and followed the expected normal contours of the abdominal wall. The mesh length was measured at the marked points of the defect edge on the same CT image.

In addition, polyester mesh (Parietex Composite) was subjected to failure testing by a Zwick-Roell Z2.0 machine (Zwick-Roell, Ulm, Germany). The mesh was cut into $140 \mathrm{~mm}$ by $25 \mathrm{~mm}$ specimens in the longitudinal (warp) and transverse (weft) direction. Specimens were hydrated in saline at 37 degrees Celsius for at least 10 min before testing. Mesh was placed between grips of the machine with $20 \mathrm{~mm}$ overlap on each side. The mesh was then subjected to loading at a rate of $10 \mathrm{~mm}$ per minute. These settings were chosen in accordance with the British Standards Institute. Each test was ended when the mesh ruptured.

\section{Statistics}

Data were expressed as mean and standard deviation where appropriate. Parametric data was compared using a $T$ test while nonparametric data were analysed using a Chi squared test. Analysis was performed using IBM Statistics for windows, version 22.0 (Armonk New York USA, IBM corp.)

\section{Results}

Thirty-two of the 100 patients evaluated for ventral hernia repair were considered suitable for a laparoscopic approach. These patients were significantly older than their open counterparts but demographic data were otherwise similar (Table 1). The average defect width was $6.2 \mathrm{~cm}$ (range $2-12 \mathrm{~cm}$ ) while the average mesh used to cover this defect was $17 \mathrm{~cm}$ (range 15-25 cm). There were no intraoperative complications with this group of patients while the average hospital stay was 2 days. Four patients had a seroma while one had severe pain postoperatively.

Electronic records of patients were reviewed at a mean follow-up of 34 months (range 24-48 months) and again at a mean follow-up of 104 months (range 92-116 months). Nineteen (59\%) of the 32 patients operated on had an abdominopelvic CT on follow-up, the indications for CT are shown on Table 2. At 34 months no recurrences were recorded while at 104 months, three $(9.4 \%)$ patients were noted to have a recurrence on CT scan. Two of these were noted on staging $\mathrm{CT}$ for cancer, while one presented acutely with incarcerated omentum in a hernia sac. The interval between operation and recurrence was 81,90 and

Table 1 Demographic details of patients undergoing laparoscopic and open ventral hernia repair

\begin{tabular}{llll}
\hline & $\begin{array}{l}\text { Laparoscopic } \\
(N=32)\end{array}$ & $\begin{array}{l}\text { Open } \\
(N=68)\end{array}$ & $p$ value \\
\hline Age years & $63.5(11.5)$ & $53.7(15.5)$ & 0.001 \\
Male/female & $10 / 22$ & $33 / 35$ & 0.158 \\
BMI & $31.7(4.9)$ & $32.2(6.9)$ & 0.318 \\
ASA score & $2.39(0.8)$ & $2.21(0.62)$ & 0.158 \\
Incisional hernia* & 27 & 59 & 0.750 \\
\hline
\end{tabular}

Data area expressed as mean (standard deviation) where appropriate *Others included umbilical (5), epigastric (5), Spigelian (2) and port site hernia (2) 
Table 2 Indications for CT scan

\begin{tabular}{lc}
\hline Indication & Number \\
\hline Cancer* & 10 \\
Pain & 5 \\
Sepsis & 2 \\
Obstruction $^{\#}$ & 1 \\
Incarcerated hernia $^{*}$ & 1 \\
\hline
\end{tabular}

*This included diagnosis, staging or follow-up of a patient with cancer

\#This patient had adhesive obstruction which settled on conservative treatment

116 months, respectively. Two of the recurrences involved central rupture of the mesh while the third was observed between two, tacks lateral to the original defect. An additional patient had a large pelvic cancer which ruptured through the mesh 47 months after her hernia repair.

\section{Elasticity of mesh}

Ten meshes were tested -5 in the longitudinal direction and 5 in the transverse direction. Mesh lost its elasticity at a force of $1.8 \mathrm{~N} / \mathrm{cm}$ in the longitudinal direction and $3.2 \mathrm{~N} / \mathrm{cm}$ in the transverse direction. This was associated with an increase in length of 22 and $5 \mathrm{~mm}$, respectively. Mesh ruptured at a force of $20.8 \mathrm{n} / \mathrm{cm}$ in the longitudinal direction and $42.1 \mathrm{~N} /$ $\mathrm{cm}$ in the transverse direction. The increase in length associated with this was 79 and $36 \mathrm{~mm}$, respectively (Table 3).

In nine $(47 \%)$ of patients who had a CT, the mesh had stretched across the defect by an average of $21.3 \%$ (range 5.7-40\%). This was observed for both small and large defects-Figs. 1 and 2.

\section{Discussion}

This study shows that 3 (9.4\%) of 32 patients had a late recurrence of their hernia following laparoscopic repair of a ventral hernia using a polyester mesh. Recurrences were only observed after 5 years of follow-up. This pattern of recurrence has not been reported before and highlights the importance of long-term follow-up in this group of

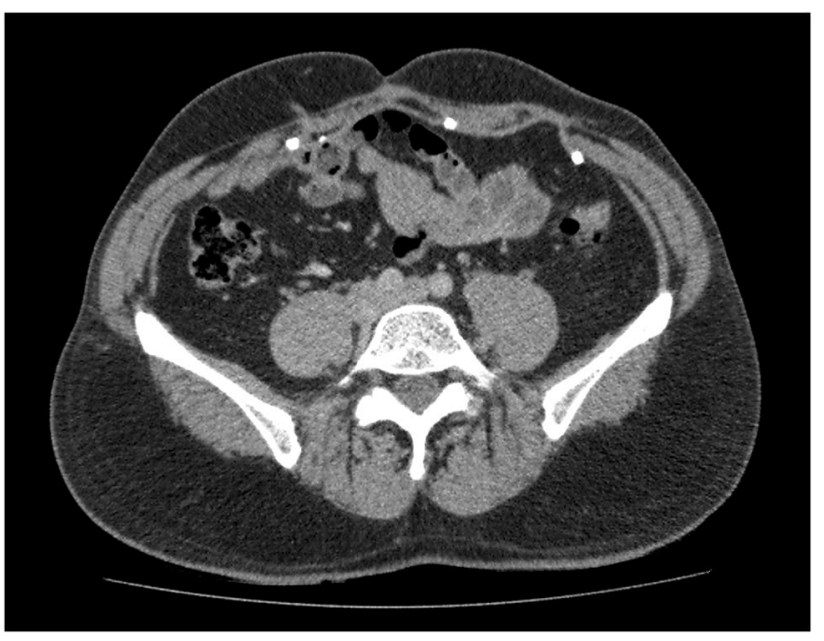

Fig. 1 Mesh has stretched by $40 \%$ across a $2.5-\mathrm{cm}$ defect on the patient's left side 2 years after hernia repair. Note the mesh is also stretched across a midline defect but to a lesser degree

patients. Around $50 \%$ of recurrences for both open and laparoscopic ventral hernia are thought to happen within 2 years of operation, yet in this study none were recorded at that time point [4].

A possible explanation for hernia recurrence particularly after bridging a defect could be loss of elasticity of the mesh with herniation of the mesh into the defect. MRI studies have shown that an intraabdominal pressure of 18.6 Kpa generates a force on the abdominal wall of $28 \mathrm{n} /$ $\mathrm{cm}$ in the transverse direction and $22 \mathrm{n} / \mathrm{cm}$ in the longitudinal direction [5]. This pressure can be generated by coughing or jumping in a healthy adult and far outstrips the force required to convert the mesh used in this study from elastic to plastic [6].

Although CT scans confirmed stretching of the polyester mesh used to bridge the defect, this was not present in all patients and in some it was minor and not clinically relevant. Degradation of the mesh over time, combined with stretching, seems a more logical explanation of the late recurrence seen in our study. A study by Riepe et al. examining the in vivo hydrolysis of polyester vascular grafts demonstrated that hydrolytic degradation of polyester reduced their bursting pressure by $31.4 \%$ at 10 years [7]. Degradation is obviously observed for all mesh products
Table 3 Mechanical properties of polyester mesh

\begin{tabular}{lllll}
\hline Mesh direction & $\begin{array}{l}\text { Loss of elasticity } \\
(\mathrm{N} / \mathrm{cm})^{*}\end{array}$ & $\begin{array}{l}\text { Increase in length } \\
(\%)\end{array}$ & Rupture (N/cm)* & $\begin{array}{l}\text { Increase in } \\
\text { length (\%) }\end{array}$ \\
\hline Longitudinal (SD) & $1.8(0.1)$ & $22(2)$ & $20.8(1.6)$ & $79(3)$ \\
Transverse (SD) & $3.2(0.2)$ & $5(0)$ & $42.1(3.6)$ & $36(3)$ \\
\hline
\end{tabular}

Data are expressed as mean (standard deviation)

*Force (N/cm): Newton per centimetre 


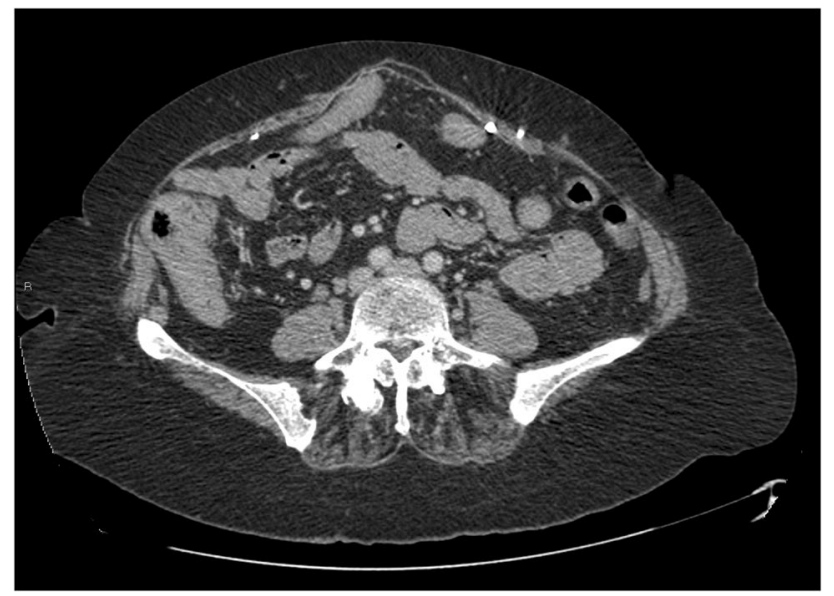

Fig. 2 Mesh has stretched by $24 \%$ across a $6.5-\mathrm{cm}$ midline defect 4 years after hernia repair

and is likely to weaken the mesh over time so that rupture force is considerably less than it was de novo [8-10].

Differential outcome for different meshes have been observed following both open and laparoscopic ventral hernia repair $[2,4,11]$. Generally lightweight meshes break and give rise to early recurrence. There is some evidence that suturing a defect rather than just bridging it reduces early recurrence [1]. However, closing the defect was not performed in this study and yet all recurrences were seen after 5 years.

One of the drawbacks of this study is the lack of longterm clinical follow-up. However, in the current climate of Covid-19 this would not be possible. An alternative is to look at recurrence through well-kept national registries. This identifies patients that undergo reoperation for their hernia and if we were to do that only 1 (3\%) of the recurrences in our study would have been found. As many patients require a CT scan for one reason or another as they get older, while not perfect, this will identify the patient who is asymptomatic or does not wish a further operation for their hernia. Scotland has a national linked picture archiving and communication system (PACS) which is in effect a comprehensive imaging registry for our population.

A further drawback of this study is that the number of patients in the study was small and represented only one-third of those referred for operation over the 2-year period. However, all the operations were performed by an experienced laparoscopic surgeon with a major interest in hernia management. The unit acted as a tertiary referral centre for complex hernias with 18 of the patients having contaminated wounds or dirty wounds secondary to infected mesh or fistulae while the remainder had complex often recurrent hernias suitable for retro-muscular or component separation repair only.

\section{Conclusion}

This study shows that late recurrence is a problem following laparoscopic ventral hernia repair with polyester mesh. The mesh loses it elasticity at a low force. This combined with degradation of polyester mesh seems the most likely cause of failure. This is unlikely to be a unique problem of polyester mesh and further long-term studies are required to better assess this operative approach to ventral hernia repair.

Acknowledgements We would like to thank Nicholas Dodds for his help with testing the mesh at the Department of Engineering, University of Glasgow. We would also like to thank Covidien for their kind donation of mesh for the mechanical failure testing study.

Funding No funding.

Availability of data and material Available if requested.

Code availability Not applicable.

\section{Declarations}

Conflict of interest Nil to declare.

Ethics approval IRB review waived: this is a retrospective audit of outcomes of patients assessed for suitability for incisional hernia repair.

Human and animal rights statement No human or animal experiments were performed in this study.

Consent for publication All authors consent to publication.

Open Access This article is licensed under a Creative Commons Attribution 4.0 International License, which permits use, sharing, adaptation, distribution and reproduction in any medium or format, as long as you give appropriate credit to the original author(s) and the source, provide a link to the Creative Commons licence, and indicate if changes were made. The images or other third party material in this article are included in the article's Creative Commons licence, unless indicated otherwise in a credit line to the material. If material is not included in the article's Creative Commons licence and your intended use is not permitted by statutory regulation or exceeds the permitted use, you will need to obtain permission directly from the copyright holder. To view a copy of this licence, visit http://creativecommons.org/licenses/by/4.0/.

\section{References}

1. Henrikson NA, Friis-Andersen H, Jorgensen LN, Helgstrand F, Danish Hernia Database (2021) Open versus laparoscopic incisional hernia repair: nationwide database study. BJS Open 5:zra010 
2. Petro CC, Nahabet EH, Criss CN et al (2015) Central failures of lightweight monofilament polyester mesh causing hernia recurrence: a cautionary note. Hernia 19:155-159

3. Totten C, Becker P, Lourd M, Ssott RJ (2019) Polyester versus polypropylene, do mesh materials matter? A meta-analysis and systematic review. Med Devices Evid Res 2019:369-378

4. Kocherling F (2019) Recurrent Incisional hernia repair-an overview. Front Surg 6:26

5. Forstemann T, Trzewik J, Holste J, Batke B, Konering MA, Wolloscheck T, Hartung C (2011) Forces and deformations of the abdominal wall-a mechanical and geometric approach to the linea alba. J Biomed 44(4):600-606

6. Cobb WS, Burns JM, Kercher KW, Matthews BD, James Norton H, Todd HB (2005) Normal intraabdominal pressure in healthy adults. J Surg Res 129(2):231-235. https://doi.org/10.1016/j.jss. 2005.06.015

7. Klinge U, Klosterhalfen B (2012) Modified classification of surgical meshes for hernia repair based on the analyses of 1,000 explanted meshes. Hernia 16(3):251-258. https://doi.org/10.1007/ s10029-012-0913-6

8. Wood AJ, Cozad MJ, Grant DA, Ostdiek AM, Bachman SL, Grant SA (2013) Materials characterization and histological analysis of explanted polypropylene, PTFE, and PET hernia meshes from an individual patient. J Mater Sci Mater Med 24(4):1113-1122. https://doi.org/10.1007/s10856-013-4872-y

9. Deeken CR, Lake SP (2017) Mechanical properties of the abdominal wall and biomaterials utilized for hernia repair. J Mech Behav Biomed Mater 74:411-427. https://doi.org/10.1016/j.jmbbm. 2017.05.008

10. Riepe G, Loos G, Imig H, Schroder A, Schneider E, Petermann J, Rogge A et al (1997) Long term in vivo alterations of polyester vascular grants in humans. Eur J Vasc Endovasc Surg 13:540-548

11. Helgstrand F, Thygesen LC, Bisgaard T, Jorgensen LN, FriisAndersen H (2020) Differential recurrence after laparoscopic incisional hernia repair: importance of a nationwide registry-based mesh surveillance. BJS 107:1130-1136

Publisher's Note Springer Nature remains neutral with regard to jurisdictional claims in published maps and institutional affiliations. 